Running Head: Contextual cuing without attentional guidance

IN PRESS JEP:HPP (Aug, 2020)

\title{
There is More to Contextual Cuing than Meets the Eye: Improving Visual Search without Attentional Guidance towards Predictable Target Locations
}

\author{
Miguel A. Vadillo ${ }^{1}$, Tamara Giménez-Fernández ${ }^{1}$, \\ Tom Beesley², David R. Shanks ${ }^{3}$, David Luque ${ }^{1,4}$ \\ ${ }^{1}$ Departamento de Psicología Básica, Universidad Autónoma de Madrid, Spain \\ ${ }^{2}$ Department of Psychology, Lancaster University, $U K$ \\ ${ }^{3}$ Department of Experimental Psychology, University College London, UK \\ ${ }^{4}$ Departamento de Psicología Básica, Universidad de Málaga, Spain
}

\section{Wordcount (main text): 2,493}

Mailing address:

Miguel A. Vadillo

Departamento de Psicología Básica

Facultad de Psicología

Universidad Autónoma de Madrid

28049 Madrid, Spain

e-mail: miguel.vadillo@uam.es

Autor note: MV was supported by grant PSI2017-85159-P (AEI / FEDER, UE). MV and TGF were supported by grant 2016-T1/SOC-1395 (Comunidad de Madrid, Programa de Atracción de Talento Investigador). MV and DS were supported by grant ES/P009522/1 from the Economic and Social Research Council. DL was supported by grant PGC2018094694-B-I00 (AEI / FEDER, UE) and grant 2017-T1/SOC-5147 (Comunidad de Madrid, Programa de Atracción de Talento Investigador). Correspondence concerning this article should be addressed to Miguel A. Vadillo, Departamento de Psicología Básica, Facultad de Psicología, Universidad Autónoma de Madrid, 28049 Madrid, Spain. E-mail: miguel.vadillo@uam.es 


\begin{abstract}
It is usually easier to find objects in a visual scene as we gain familiarity with it. Two decades of research on contextual cuing of visual search show that repeated exposure to a search display can facilitate the detection of targets that appear at predictable locations in that display. Typical accounts for this effect attribute an essential role to learned associations between the target and other stimuli in the search display. These associations improve visual search either by driving attention towards the usual location of the target or by facilitating its recognition. Contrary to this view, we show that a robust contextual cuing effect can also be observed when repeated search displays do not allow the location of the target to be predicted. These results suggest that, in addition to the mechanisms already explored by previous research, participants learn to ignore the locations usually occupied by distractors, which in turn facilitates the detection of targets even when they appear in unpredictable locations.
\end{abstract}

\title{
Public Significance Statement
}

Over the last two decades, the contextual cuing effect has become a popular laboratory model to explore the interconnection between learning, memory, and attention. In this task, participants' ability to find a target in a search display improves as a result of repeated exposure to the same search display. The most common explanation for this effect is that participants learn to use contextual information to predict the target's location. Contrary to this view, we show that a reliable contextual cuing effect can be found even in situations where the location of the target is completely unpredictable.

Keywords: Attentional guidance; Associative learning; Contextual cuing; Distractor suppression; Visual search. 
Objects are easier to find and identify when presented in a familiar and consistent context (Biederman, 1972). Experimental psychologists have devised numerous experimental tasks aimed at unravelling the cognitive processes that allow us to exploit these kinds of statistical regularities in visual input (Schapiro \& Turk-Browne, 2015). The contextual cuing task, in particular, has become a popular means to explore how visual search is guided by consistent patterns of contextual information (Chun \& Jiang, 1998). In a typical experiment, participants are asked to find a T-shaped target among a number of L-shaped distractors. Identical search displays are presented repeatedly over the experiment, although participants are not told this. Eventually, they become faster at finding the target in these repeated displays than in completely new (or random) displays, suggesting that, beyond becoming familiar with the visual search task, they are also able to encode information about those specific search displays.

The search advantage for repeated patterns seems to be largely driven by an improvement in attentional guidance towards the usual location of the target. For instance, participants make fewer fixations in repeated search displays than in new displays (Beesley et al., 2018; Peterson \& Kramer, 2001). Similarly, search slopes (i.e., the average amount of time needed to find the target relative to the number of elements in the search display) are usually shallower for repeated than for new search displays (Chun \& Jiang, 1998), suggesting that visual search is more efficient in the former. In addition to visual guidance, it has been suggested that late processes taking place once the target has been selected might also play a role in contextual cuing. For instance, it is possible that, once fixated, it is easier to identify the target or to respond to it when it appears in a predictable and familiar location (Kunar et al., 2007). However, the empirical support for the role of response selection is much weaker than for attentional guidance (Sisk et al., 2019).

In any event, both mechanisms, attentional guidance and response selection, hinge on the existence of an association between the repeated configuration of distractors and the location of the target, either because the configuration drives attention towards the target or because the distractors immediately surrounding the target facilitate its recognition or the response it elicits. The possibility that mechanisms other than distractor-target associations contribute to contextual cuing is usually dismissed (e.g., Sisk et al., 2019) based on the results of an early study by Chun and Jiang (1998, Experiment 3), who observed that contextual cuing was absent if each repeated display was paired with a different target location across repetitions. Contextual cuing seems to depend critically, this evidence 
suggests, on the fact that the location of the target can be predicted on the basis of the distractors.

Other studies, in contrast, suggest that contextual cuing can also take place in situations where the exact location of the target is unpredictable. For instance, Kunar and Wolfe (2011) and Wang et al. (in press) found that a single repeated search display can be associated with two, three or four different target locations. Beesley et al. (2015, Experiment 1) failed to find significant evidence of cuing when each repeated search display was paired with four different target locations. However, there was a small $\left(d_{z}=\right.$ 0.24) trend in the direction of a contextual cuing effect. Most importantly, in several unpublished experiments that followed up on this result (see Experiments S1-S4 in the Supplementary Material) we did find significant evidence of cuing under those conditions. One of these studies (Experiment S3) revealed significant cuing even when the target appeared in completely random locations on the screen across repetitions of the search display.

These results speak against the assumption that contextual cuing depends exclusively on associations between the configuration of distractors and the location of the target, but they are consistent with the predictions of an influential model of contextual cuing developed by Brady and Chun (2007). In this model, contextual cuing not only involves learning to predict the location of the target; it also requires learning to ignore the locations usually occupied by distractors within a repeated search display. This is achieved by the gradual development of inhibitory associations linking all distractors with each other (for simulations, see Beesley et al., 2015). If follows from this model that the mere repetition of a search display should, on its own, result in some degree of contextual cuing, even if the location of the target is completely unpredictable.

The present study was designed to test this prediction with larger samples and additional controls missing in Beesley et al. (2015) and in our unpublished Experiments S1-S4. In both Experiments 1 and 2, some displays (new) comprised unique patterns of distractors, together with targets at random or pseudo-random locations. These new neverrepeated displays comprise the baseline against which reaction times in the conditions of interest were compared. These conditions (repeated-4 and repeated-R in Experiments 1 and 2 , respectively) also involved random or pseudo-random target locations, but with stable patterns of distractors. Any evidence of faster responses in these conditions compared to the baseline new condition is evidence of learning not mediated by associations between a distractor configuration and the target location. Experiment 1 also included a standard 
contextual cuing condition (repeated-1), comprising repeated presentations of a given distractor display with a consistent target location, in which learning such associations was possible.

\section{Method}

\section{Participants}

A meta-analysis of Experiments S1-S4 and Experiment 1 from Beesley et al. (2015) yielded an average cuing effect of $d_{\mathrm{z}}=0.49$. An a priori power analysis showed that at least 57 participants are needed to replicate this effect in a two-tailed repeated-samples $t$ test with $\alpha=.05$ and power $=.95$. Therefore, we decided to test at least 60 participants in Experiments 1 and 2. In practice, due to the lack of precise control over the participant recruitment system at UAM, we tested 63 participants (54 female) in Experiment 1 and 81 participants (64 female) in Experiment 2. Their mean age was $19.47(S D=1.02)$ and 19.54 (1.32), respectively. All the participants were undergraduate psychology students at UAM and received academic credit for taking part in the experiment. The studies were approved by UAM's ethics committee (CEI-80-1473). All participants signed an informed-consent form.

\section{Stimuli}

Search displays consisted of 16 distractors and a target, positioned in a $12 \times 12$ grid, invisible to participants. Each distractor was an L-shaped stimulus that could be rotated $0^{\circ}$, $90^{\circ}, 180^{\circ}$, or $270^{\circ}$. Targets were T-shaped stimuli rotated either $90^{\circ}$ or $270^{\circ}$. Distractors and targets subtended approximately $0.8^{\circ}$ at a viewing distance of $60 \mathrm{~cm}$ and could appear in red, green, yellow, or blue, presented against a gray background.

\section{Procedure and Design}

Each trial began with a 1-sec white fixation cross, followed by the search display. Participants were instructed to search for the T and press key " $\mathrm{z}$ " if the stem of the T pointed to the left and " $\mathrm{m}$ " if it pointed to the right. They were instructed to find the target as quickly as possible but without making errors. Incorrect responses were immediately followed by a 2-sec "Error!" message. A 1-sec blank screen was presented before the onset of the following trial. Participants were allowed to take a 20 -sec rest break every 120 trials. 
Both experiments comprised 30 blocks of trials. In Experiment 1, each block included 12 trials. Four trials in each block belonged to the repeated-1 condition, where all the elements of the displays, including the target, appeared in the same location and color across repetitions. Each of the four trials featured a different search display, with a different target location. Four trials per block belonged to the new condition. In this case, all the distractors appeared in random locations, different across repetitions. The targets, however, always appeared in one of the four locations reserved for that purpose. Four trials per block belonged to the repeated-4 condition. In this case, the configuration of distractors within the search display was kept identical across repetitions, as in condition repeated-1, but the location of the target changed from one repetition to the next. The assignment of target locations to search displays in this condition ensured that the same display could not contain the target in the same location again until it had already been paired with the other three reserved locations in subsequent blocks. Different target locations were used in each condition, always ensuring that the four search displays included in each condition presented the target in different quadrants.

In Experiment 2, each block contained eight trials, half of them in the new condition and the other half in the repeated-R condition. The search displays in the new condition were constructed as in Experiment 1, with the only exception that no locations were preselected to contain the target, which could appear anywhere in the display. In condition repeated-R, the configuration of distractors in the search displays remained identical over repetitions, but the target could appear in any random location.

\section{Results}

As in Experiments S1-S4, only participants with a mean accuracy above $95 \%$ were considered in the analyses. This led to the exclusion of three participants in Experiment 1 and three in Experiment 2. Trials with incorrect responses, trials immediately following a rest break, and trials with reaction times (RTs) longer than 10 secs were removed from the analysis. We also removed RTs 3 standard deviations above or below each participant's mean. Data from adjacent blocks were combined into 2-block epochs. Figure 1 shows mean RTs across epochs for each condition and experiment. 

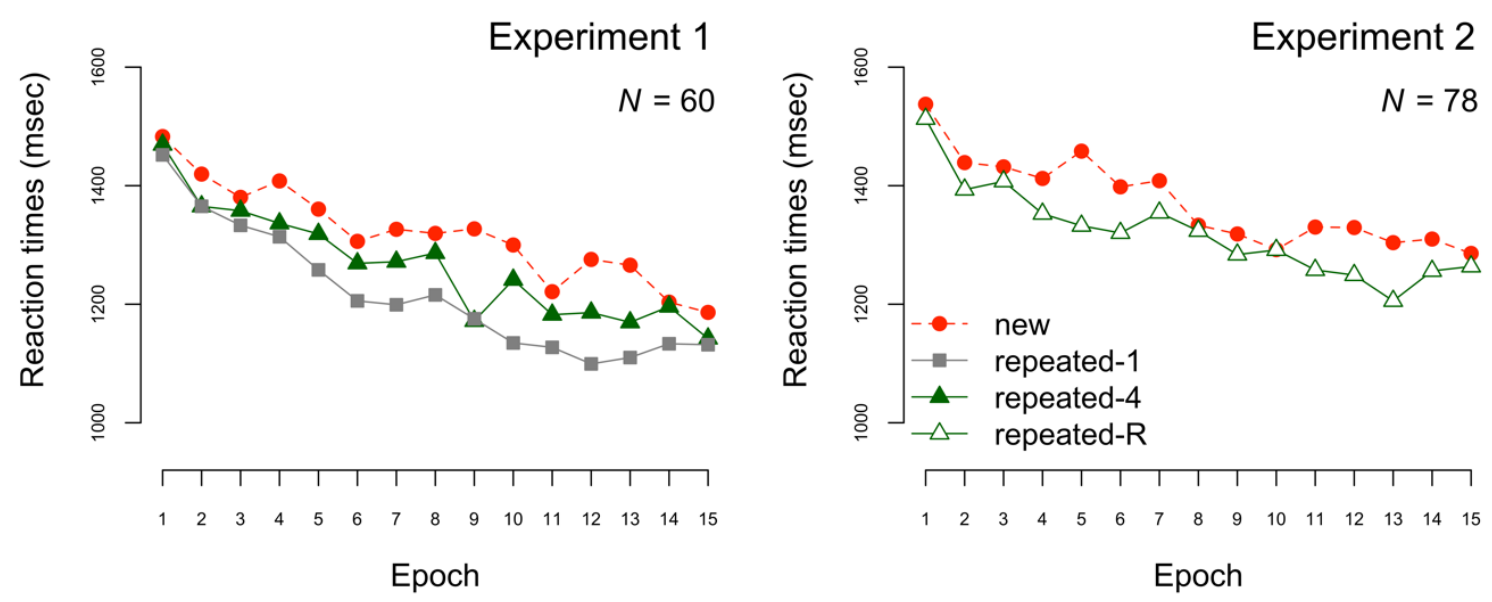

Figure 1. Reaction times (in milliseconds) in Experiments 1 and 2.

RTs from both experiments were analyzed with repeated-measures analyses of variance with Condition and Epoch as factors. In Experiment 1, the ANOVA yielded significant main effects of Condition, $F(2,118)=21.99, p<.001, \eta_{p}^{2}=.27$, and Epoch, $F(14,826)=38.31, p<.001, \eta_{p}^{2}=.39$, and a significant Condition $\times$ Epoch interaction, $F(28,1652)=1.71, p=.012, \eta_{p}^{2}=.03$. As shown in Figure 1, RTs were faster in condition repeated- 1 than in condition repeated- $4, t(59)=2.67, p=.010, d_{\mathrm{z}}=0.34$, and they were also faster in condition repeated-4 than in condition new, $t(59)=3.90, p<.001, d_{z}=0.50$. The analysis of Experiment 2 yielded significant effects of Condition, $F(1,77)=24.58, p<$ $.001, \eta_{p}^{2}=.24$, and Epoch, $F(14,1078)=12.87, p<.001, \eta_{p}^{2}=.14$, but no interaction between them, $F<1$.

\section{Discussion}

The results of the present experiments show that contextual cuing can occur even when the location of the target is completely unpredictable. In Figure 2 we present the results of a meta-analysis collating Experiments 1 and 2 with Experiment 1 from Beesley et al. (2015), Experiments S1-S4 (reported in the Supplementary Materials), and an experiment by Zhao (2014) conducted under the supervision of one of us (T. Beesley). Collectively, these eight experiments represent the full record of studies on this effect conducted or supervised by the authors of the present article. The meta-analysis finds an overall effect of $d_{z}=0.60,95 \%$ confidence interval [0.42, 0.78]. All of the experiments include this mean effect size within the upper bound of their individual confidence intervals. This effect is significant but admittedly smaller than the standard contextual 
cuing effect $\left(d_{\mathrm{z}}=0.97\right.$, for the comparison between repeated- 1 and new conditions in Experiment 1), which might explain why not all previous attempts to detect it have yielded significant results. For instance, with just 10 participants, the seminal Experiment 3 by Chun and Jiang (1998) barely achieves 55\% power to detect an effect of this size.

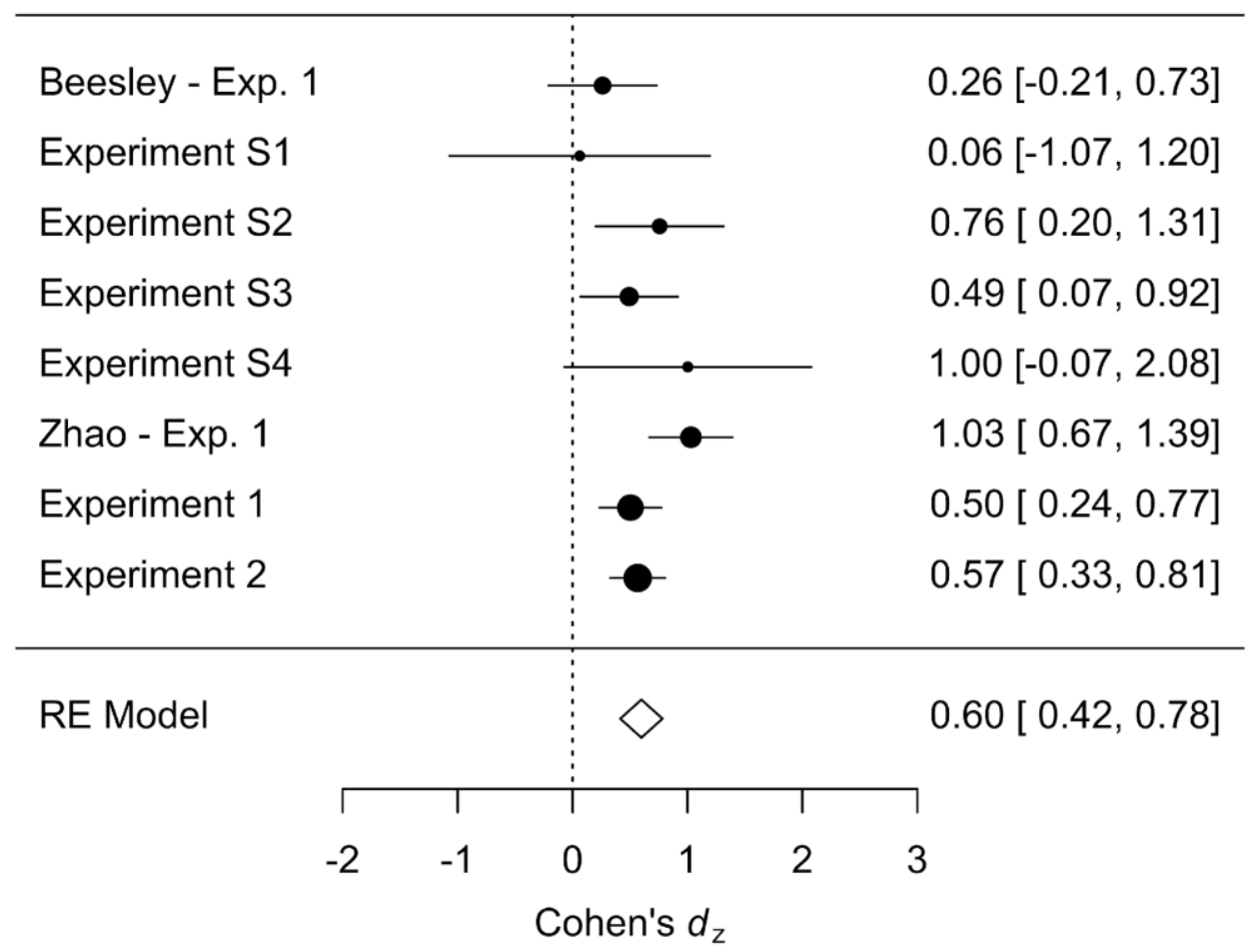

Figure 2. Forest plot and meta-analysis. All the effect sizes refer to the contrast between search times for new search displays and for repeated-4 or repeated-R search displays. Error bars represent $95 \%$ confidence intervals. Heterogeneity across studies did not reach statistical significance, $Q(7)=9.96, p=.191, \tau^{2}=0.02$, $I^{2}=33.12 \%$.

As explained in the introduction, the fact that a small but reliable contextual cuing effect can be observed when repeated patterns are paired with four different target locations (Experiment 1) is not a novel finding. The same result has been reported in previous studies (Kunar \& Wolfe, 2011; Wang et al., in press) and, taken alone, does not pose a critical challenge to the hypothesis that contextual cuing is driven by associations between the configuration of distractors and the location of the target. After all, participants in these experiments were exposed to a few presentations of the same configuration with the same target location. For instance, in our Experiment 1, each repeated display was paired 7-8 times with each of the four target locations. Zellin et al. (2011) have argued that these instances of contextual cuing could be driven by learning of 
perhaps only one target location for each repeated search display, resulting in a somewhat smaller effect. However, it is difficult to apply this logic to the results of Experiment 2 (and Experiment S3 in the Supplementary Material), where the location of the target was completely random.

These results suggest that in addition to any association between distractors and targets, there are probably other mechanisms that also contribute to the search advantage for repeated patterns. Consistent with the predictions of Brady and Chun (2007), repeated exposure to search displays may not only allow participants to anticipate the most likely location of the target, but also to infer that the locations usually occupied by distractors are unlikely to contain the target and can be ignored (for simulations, see the Supplementary Matrial). Pairing the same configuration of distractors with different target locations, as we did in the present experiments, precludes the first type of learning, but not the second. This interpretation is consistent with evidence showing that the search advantage for repeated displays sometimes remains even when the location of the target changes from one stage of the experiment to another (Luque et al., 2017; Preuschhof et al., 2019). It is also consistent with additional research showing that people can learn to anticipate the location of visual distractors and suppress their processing (Failing \& Theeuwes, in press).

The model proposed by Brady and Chun (2007) assumes that the standard contextual cuing effect is driven both by distractor suppression and by learned associations between repeated search displays and target locations. This dual mechanism would explain why contextual cuing was substantially larger in condition repeated-1, which essentially replicated the standard procedure, than in conditions repeated- 4 and repeated-R, with variable target locations. It is not impossible, though, that contextual cuing is driven exclusively by one of these mechanisms or the other depending on details of the experimental procedure. Neurophysiological evidence suggests that repeated and new search displays elicit different patterns of brain activity as early as $100 \mathrm{msec}$ after stimulus onset and that these early components already encode information about the location of the target (Chaumon et al., 2008; Zinchenko et al., in press). Experimental procedures that preclude the formation of robust associations between distractor configurations and a specific target location may hinder the natural course of these early processes, resulting in a qualitatively different type of contextual cuing, driven by distractor suppression. Future research should address this possibility.

\section{Open Practices Statement}


The materials, datafiles and analysis scripts are available at https://osf.io/rmcuh/.

\section{References}

Beesley, T., Hanafi, G., Vadillo, M. A., Shanks, D. R., \& Livesey, E. J. (2018). Overt attention in contextual cuing of visual search is driven by the attentional set, but not by the predictiveness of distractors. Journal of Experimental Psychology: Learning, Memory, \& Cognition, 44, 707-21.

Beesley, T., Vadillo, M. A., Pearson, D., \& Shanks, D. R. (2015). Pre-exposure of repeated search configurations facilitates subsequent contextual cuing of visual search. Journal of Experimental Psychology: Learning, Memory, and Cognition, 41, 348362.

Biederman, I. (1972). Perceiving real-world scenes. Science, 177(4043), 77-80.

Brady, T. F., \& Chun, M. M. (2007). Spatial constraints on learning in visual search: Modeling contextual cuing. Journal of Experimental Psychology: Human Perception and Performance, 33, 798-815.

Chaumon, M., Drouet, V., \& Tallon-Baudry, C. (2008). Unconscious associative memory affects visual processing before $100 \mathrm{~ms}$. Journal of Vision, 8:10.

Chun, M. M., \& Jiang, Y. (1998). Contextual cueing: Implicit learning and memory of visual context guides spatial attention. Cognitive Psychology, 36, 28-71.

Failing, M., \& Theeuwes, J. (in press). More capture, more suppression: Distractor suppression due to statistical regularities is determined by the magnitude of attentional capture. Psychonomic Bulletin \& Review.

Kunar, M. A., Flusberg, S., Horowitz, T. S., \& Wolfe, J. M. (2007). Does contextual cuing guide the deployment of attention? Journal of Experimental Psychology: Human Perception and Performance, 33, 816-828.

Kunar, M. A., \& Wolfe, J. M. (2011). Target absent trials in configural contextual cuing. Attention, Perception \& Psychophysics, 73, 2077-2091.

Luque, D., Vadillo, M. A., López, F. J., Alonso, R., \& Shanks, D. R. (2017). Testing the controllability of contextual cuing of visual search. Scientific Reports, 7:39645.

Peterson, M. S., \& Kramer, A. F. (2001). Attentional guidance of the eyes by contextual information and abrupt onsets. Perception \& Psychophysics, 63, 1239-1249.

Preuschhof, C., Sharifan, F., Rosenblum, L., Pohl, T. M., \& Pollmann, S. (2019). Contextual cueing in older adults: Slow initial learning but flexible use of distractor configurations. Visual Cognition, 27, 563-575. 
Schapiro, A., \& Turk-Browne, N. (2015). Statistical learning. In A. W. Toga (Ed.), Brain mapping: An encyclopedic reference (Vol. 3, pp. 501-506). Academic Press:

Elsevier.

Sisk, C. A., Remington, R. W., \& Jiang, Y. V. (2019). Mechanisms of contextual cueing: A tutorial review. Attention, Perception, \& Psychophysics, 81, 2571-2589.

Wang, C., Bai, X., Haponenko, H., Hui, Y., Song, C., Zhao, G., Milliken, B., \& Sun, H.-J. (in press). Learning of association between a context and multiple possible target locations in a contextual cueing paradigm. Attention, Perception, \& Psychophysics.

Zhao, H. (2014). Exploring distractor inhibition in contextual cueing: Are distractors in repeated contexts inhibited more strongly than distractors in random contexts? (Unpublished honours thesis). University of New South Wales, Sydney, Australia.

Zellin, M., Conci, M., von Mühlenen, A., \& Müller, H. J. (2011). Two (or three) is one too many: Testing the flexibility of contextual cueing with multiple target locations. Attention, Perception \& Psychophysics, 73, 2065-2076.

Zinchenko, A., Conci, M., Töllner, T., Müller, H.J. \& Geyer, T. (in press) Automatic (mis)guidance of visuo-spatial attention by acquired scene memory: evidence from an N1pc polarity reversal. Psychological Science. 